\title{
Distance-field-based Layered Manufacturing of FGM Objects
}

$$
\text { Hong-Mei ZHOU }{ }^{a, *} \text { and Zhi-Yang WANG }{ }^{b}
$$

School of Mechanical and Power Engineering, Henan Polytechnic University, JiaoZuo, China

a zhouhongmei@hpu.edu.cn, b wangzhiyang@hpu.edu.cn

Key words: Layered Manufacturing, Distance-field-based, FGM objects

\begin{abstract}
An approach of distance-field-based layered manufacturing (LM) of FGM objects is presented in this paper. Firstly, a 3D model is sliced and scanned to a series of 2D point clouds. Then, through uniformly subdividing each 2D point cloud we can compute the signed distance field of each grid points. According to the gradient property of FGM, coupled with the material distance field, a series of iso-material sub-regions for tool path planning are obtained. In this way we can systematically represent and fabricate FGM objects by a unified distance field model.
\end{abstract}

\section{Introduction}

LM has been widely explored for fabrication of FGM objects because of its advantages: maximum material utilization, less energy intensive, ability to produce complex shapes and design. In order to systemically represent, design and fabricate FGMs, a lot of former researchers proposed many solutions. Kumar et al.[1] employed the extended r-sets (include material composition $r_{m}$-sets) to model and represent FGM objects. Shin[2] presented a representation scheme for FGM objects based on the constructive representation. Yang and Qian[3] presented the design and analysis method based on B-spline, however, it depends heavily on spatial parameterizations of control points and such parameterizations are a rather troublesome task for arbitrary 3D objects. Kou et al.[4] presented feature tree-based approach to represent the material distribution. Choi et al.[5] presented a topological hierarchy-based method to LM multi-material objects, and they adopted a distance-based approach for defining the material variation. Kim et al.[6] proposed a material pixel-based process planning for LM of heterogeneous objects. As the main advantage of distance field is that complex shaped objects can be represented easily, this paper presented a distance-field-based method to systematically represent and fabricate FGM objects. In comparison with the above mentioned representation and fabrication schemes, the approach is easy and efficient, need less memory and meet the requirement of fabrication complex objects.

The representation and fabrication process is depicted in the flowchart of Fig.1. Firstly, a 3D model is sliced and scanned to produce a series of 2D point cloud. Compute the signed distance field of every layer, and discrete points are obtained through sampling the zero iso-contour of the signed distance field and interior grid points. Determine material feature according to design intent coupled with the above discrete nodes, material distance field is formed. Then, define material variation and generate iso-material composition region in every 2D slice. Finally, the iso-materials regions for tool path planning are obtained to LM.

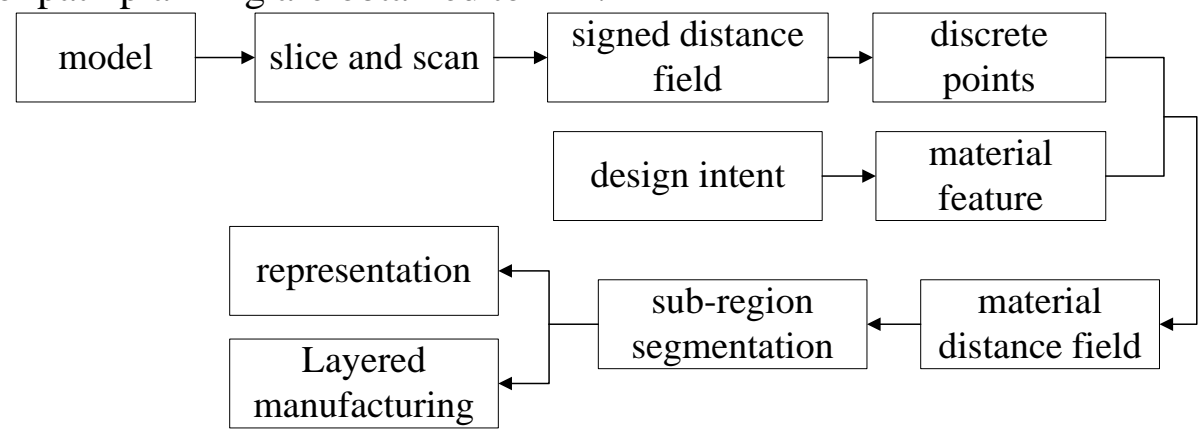

Fig.1 flowchart of distance-field-based LM of FGM objects 


\section{Geometry Signed Distance Field}

A 3D model is sliced and scanned to produce a series of 2D point cloud. For point cloud $S \in R^{2}$ of each layer in Fig.2, we establish a box bounding the point cloud, and subdivide the box with size of $N x \times N y$ (in Fig. 2 the grid size is $20 \times 20$ ). An unsigned distance field is defined as the function which calculate the distance from point $p$ (where $p$ is all grid points) to the closest point $x$ on $S$. The sign is employed to denote inside or outside the model. The unsigned distance field is quite easy to compute, and sign judgment is through node watershed algorithm [7]. All nodes are divided as three kinds: exterior node (in black), boundary node (in red) and interior node (in white), as shown in Fig.3. Then, all grid points of exterior nodes are endowed with positive, interior with negative, as shown in Fig.4 (where "+" shows that distance is positive and "o" shows that the distance is negative or zero). Sign result coupled with the distance value of every grid points, the signed distance field is showed in Fig.5.
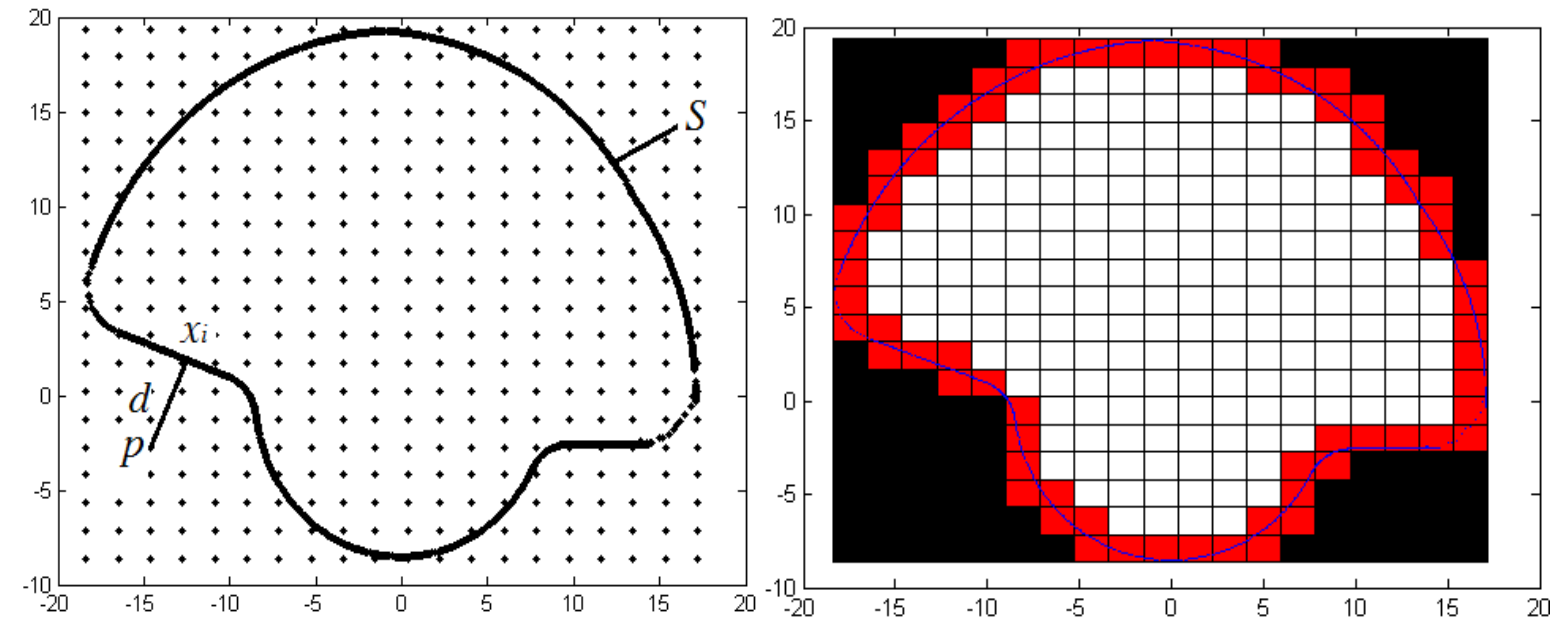

Fig.2 A bounding box (grid size 20×20) Fig.3 Exterior node, boundary node and interior node

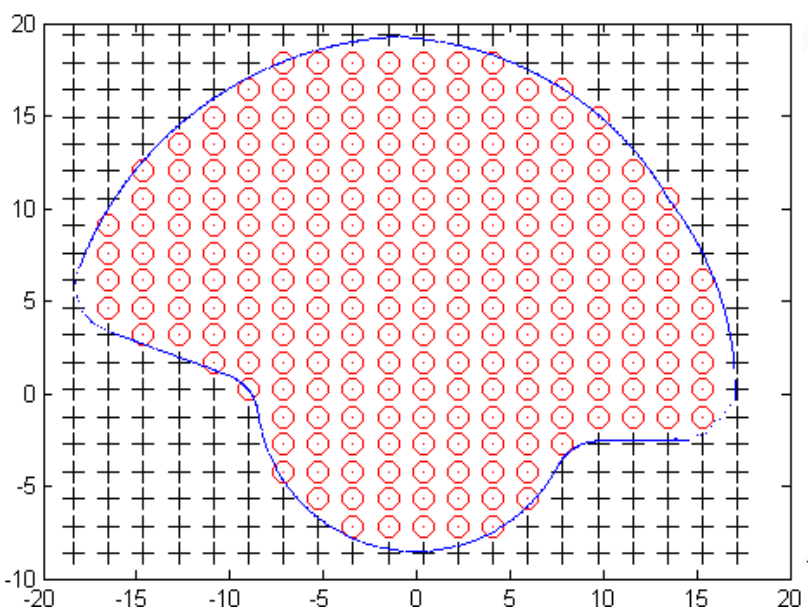

Fig.4 Sign judgment result

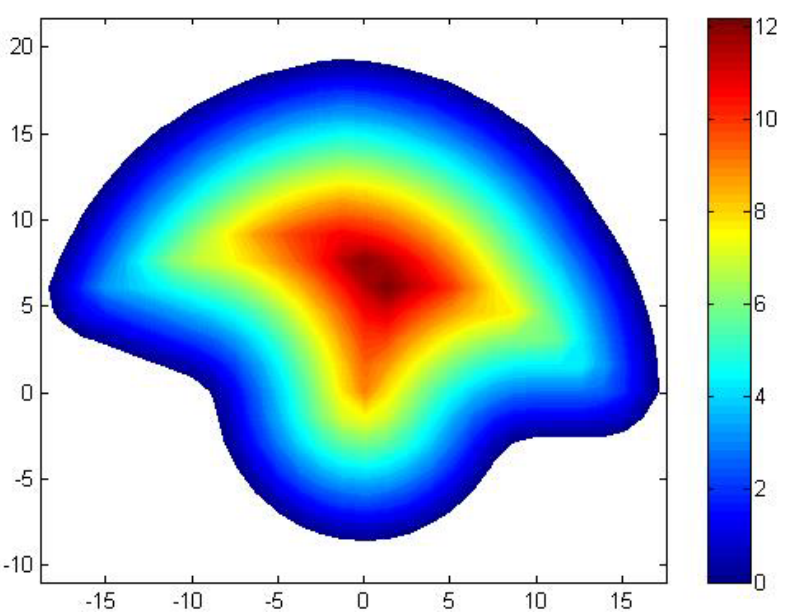

Fig.5 Signed distance field

\section{Material Distance Field Representation}

According to the FGM object property, material feature is determined by design requirements or intents as the above mentioned. FGM varies continuously in material composition as well as its performance and function. The computation of material distance field consists of two aspects: the material component, the distance value from each discrete point to material feature. The material gradient arrangement can be modified without changing the geometric distance field of the object.

If the material volume fraction is linear or non-linear gradient along the object' thickness direction, the material distribution is the function of distance field, for a two-material FGM objects: 


$$
\left.\begin{array}{c}
\text { m aterial 1: } f_{1}(d)=(\alpha d)^{k} \\
\text { m aterial 2: } f_{2}(d)=1-(\alpha d)^{k}
\end{array}\right\}
$$

Where, $d$ is normalized distance from discrete points $N$ (which are obtained through sampling the zero iso-contour of the geometry distance field and interior grid points) to model surface, $\alpha$ is the scale factor, $k$ is used to describe the gradient property, if $k$ is 1 , material property shown linear various, and which is determined according to design requirements.

In Fig. 5, $\alpha=1, k=1$, for each grid point $(i, j)$ inside of $S$, the material composition volume is:

$$
\left.\begin{array}{c}
f_{1}(d)=d_{i, j} / \max \left(d_{i, j}\right) \\
f_{2}(d)=1-d_{i, j} / \max \left(d_{i, j}\right)
\end{array}\right\}
$$

Where $d_{i, j}$ denotes the distance value from a grid point $(i, j)$ to material feature, $\max \left(d_{i, j}\right)$ denotes maximum distance value of the above distance field. In Fig.5, $\max \left(d_{i, j}\right)=11.76$ in the center position. The points on each iso-contour extracted from the signed distance field own the same material composition in Fig. 5. The discretization resolution can be varied to meet the requirement of representation and fabrication requirement.

According to the design requirement, we can have various iso-contours. If the material feature is not linear gradient along the object' thickness direction, like Fig.6, material feature is the geometry center, the material distance value of every discrete point can be computed. For each grid points inside of $S$, the material composition is: $d_{i} / \max \left(d_{m}\right) \quad(i=1, \ldots, k k)$. Here, $d_{m}$ denotes the distance value from a point of $N$ to material feature (center in Fig.6). Fig.6 lists one material volume fraction variable from 0 to 1.

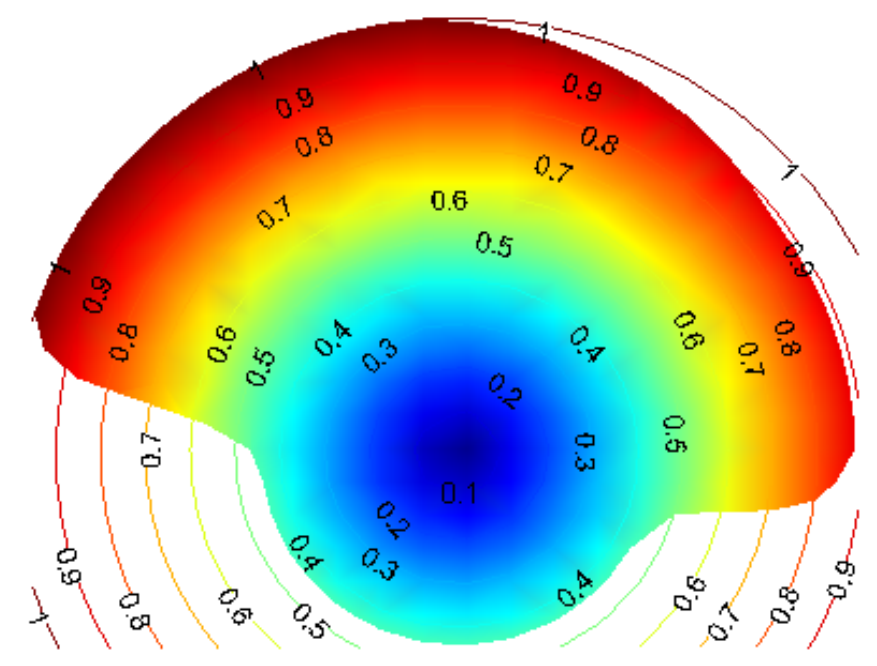

Fig.6 Material distance field 


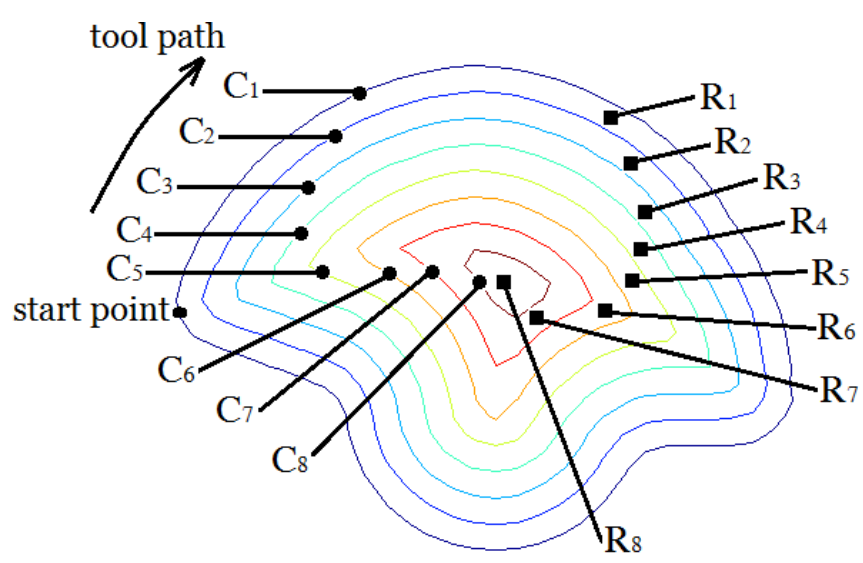

Fig. 7. Iso-contours of distance field

In order to represent colors of the distance field different primary colors are employed [8]. In this paper, the property value of a primary material is denoted by color value [R,G, B]. The property value of the two primary material $m_{1}$ and $m_{2}$ is [R1,G1,B1], [R2,G2,B2], respectively. Red RGB is 1,0,0, green RGB is 0,1,0, blue RGB is 0,0,1, black GRB is 0,0,0, white RGB is 1,1,1. In Fig.6, $[\mathrm{R} 1, \mathrm{G} 1, \mathrm{~B} 1]=\left[f_{1}(d), f_{1}(d), f_{1}(d)\right],[\mathrm{R} 2, \mathrm{G} 2, \mathrm{~B} 2]=\left[1-f_{1}(d), 1-f_{1}(d), 1-f_{1}(d)\right]$.

\section{LM of FGM Objects}

Laser-based LM is based on a layer by layer overlying approach, which can deposit different materials by inputting different material proportioning. The detail fabrication process of FGM objects is as follows:

(1) For a 3D model, as the above mentioned modeling of FGMs based on distance field, is described as:

$$
\left\{\begin{array}{c}
R^{2} \rightarrow d_{(i, j)}(S) \\
M^{n} \rightarrow d_{m(i, j)}(r)
\end{array} ; \quad(i=1, \ldots, N x ; j=1, \ldots, N y)\right.
$$

Where $d(S)$ denotes the geometry distance field, and $d_{m}(r)$ denotes the material distance field from the discrete point to material feature (r).

(2) Every layer is defined by a layer thickness $\Delta z$ and a series of iso-material contours which can be gained through extracting iso-material contour of distance field. The tool paths for material deposition will move along these iso-material contours. Fig.7 shows the iso-contours of distance field. In Fig.7, C1 C8 are a series of iso-contours of Fig.5, the distance value is 0, 1.5, 3, 4.5, 6, 7.5, 9, 10.5, respectively. Sub-region (R1 R7) are enclosed by the relative contours (C1,C2), (C2,C3), (C3,C4), (C4,C5), (C5,C6), (C6,C7), (C7,C8), respectively.

(3) We can compute the primary material volume fraction in Eq.(2). Then, according to this volume fraction the primary materials are mixed thoroughly by powder feeder, through which the mixture powder is fed to the laser molten pool. After completing a layer using the laser scanning (The start point and end point are the same one as shown in Fig.7), laser head and extrusion nozzle have the rise of $\Delta z$ in the $Z$-axis, and next to scan according to the iso-material scanning trace of the next layer.

\section{Conclusion}

This paper presented an approach for distance-field-based LM of FGM objects. The approach united the geometry distance field with material distance field to represent and fabricate FGM objects. In comparison with pixel-based, topological hierarchy-based representation schemes, the approach is easy and efficient, need less memory and meet the requirement of fabrication complex 
objects.

\section{Acknowledgement}

The work described in this paper was supported by a grant from the National Natural Science Foundation of China (Projects No.51505131, U1304523), the Fundamental Research Funds for the Universities of Henan Province (NSFRF140124), and a doctor fund (No. B2013-032) in Henan Polytechnic University. The correlative members of the projects are hereby acknowledged.

\section{Reference}

[1] Kumar V, Dutta D. J Mech Des 1998;120(4):659-667.

[2] Shin K H, Natu H, Dutta D, et al. Mater Des. 2003, 24(5) p.339-353.

[3] Yang P H, Qian X P. Computer-Aided Design. 2007, 39(2) p.95-111.

[4] Kou X Y, Tan S T. Materials and Design. 2007, 28(10) p.2549-2565.

[5] S.H.Choi, H.H.Cheung.Computers in Industry,60(2009)p.349-363.

[6] Hong Seok Kim, Ki-Hoon Shin. International journal of precision engineering and manufacturing 15 (2014) p2421-2247.

[7] H.M.Zhou,Z.G.Liu,M.X.Li,et al. Materials Science Forum Vols,628-629(2009)p.293-298.

[8] W.K.Chiu, K.M.Yu. Computer-Aided Design 40(2008) p1080-1094. 